\title{
Multidisciplinary treatment for peripheral arterial occlusive disease and the role of eHealth and mHealth
}

This article was published in the following Dove Press journal:

Journal of Multidisciplinary Healthcare

5 October 2012

Number of times this article has been viewed

\author{
Hugo JP Fokkenrood ${ }^{1,2}$ \\ Gert-Jan Lauret ${ }^{1,2}$ \\ Marc RM Scheltinga ${ }^{4}$ \\ Cor Spreeuwenberg ${ }^{3}$ \\ Rob A de Bie ${ }^{2}$ \\ Joep AW Teijink ${ }^{1,2}$ \\ 'Department of Vascular Surgery, \\ Catharina Hospital, Eindhoven, \\ The Netherlands; ${ }^{2}$ CAPHRI Research \\ School, Department of Epidemiology, \\ ${ }^{3}$ Department of Integrated Care, \\ Maastricht University, Maastricht, \\ The Netherlands; ${ }^{4}$ Department of \\ Vascular Surgery, Maxima Medical \\ Centre, Veldhoven, The Netherlands
}

Correspondence: Joep Teijink Department of Vascular Surgery, Catharina Ziekenhuis Eindhoven, Michelangelolaan 2, 5623 EJ, Eindhoven, The Netherlands Tel +3I 402396349 Email joep.teijink@cze.nl

\begin{abstract}
Increasingly unaffordable health care costs are forcing care providers to develop economically viable and efficient health care plans. Currently, only a minority of all newly diagnosed peripheral arterial occlusive disease (PAOD) patients receive efficient and structured conservative treatment for their disease. The aim of this article is to introduce an innovative effective treatment model termed ClaudicatioNet. This concept was launched in The Netherlands as a means to combat treatment shortcomings and stimulate cohesion and collaboration between stakeholders. The overall goal of ClaudicatioNet is to stimulate quality and transparency of PAOD treatment by optimizing multidisciplinary health care chains on a national level. Improved quality is based on stimulating both a theoretical and practical knowledge base, while eHealth and mHealth technologies are used to create clear insights of provided care to enhance quality control management, in addition these technologies can be used to increase patient empowerment, thereby increasing efficacy of PAOD treatment. This online community consists of a web portal with public and personal information supplemented with a mobile application. By connecting to these tools, a social community is created where patients can meet and keep in touch with fellow patients, while useful information for supervising health care professionals is provided. The ClaudicatioNet concept will likely create more efficient and cost-effective PAOD treatment by improving the quality of supervised training programs, extending possibilities and stimulating patient empowerment by using eHealth and mHealth solutions. A free market principle is introduced by introducing transparency to provided care by using objective and subjective outcome parameters. Cost-effectiveness can be achieved using supervised training programs, which may substitute for or postpone expensive invasive vascular interventions.
\end{abstract}

Keywords: intermittent claudication, multidisciplinary treatment, cost-effectiveness, eHealth

\section{Introduction}

In the near future, the increasingly unaffordable health care costs in Europe (from $13 \%$ of national income in 2012 to $31 \%$ in 2040 in The Netherlands, comparable to other European countries), necessitates a more economically viable and efficient healthcare system. ${ }^{1}$ In a recently released document titled "Healthcare, how much is it worth extra?", the Dutch Ministry of Health, Welfare and Sport notes several reasons for this increase. ${ }^{1}$ The aging population explains $25 \%$ of this rise, while a larger amount occurred due to growing prosperity including the introduction of new technologies, changing expectations of health care in society, and increasing life expectancies. Additionally, changes in epidemiologic factors, causing an increase in patients with chronic diseases, will lead to a rise in demand for health care, again resulting in increased costs. These changes in society and their influence on health care are wellreflected by peripheral arterial occlusive disease (PAOD). PAOD is a chronic systemic 
disease caused by the manifestation of atherosclerosis. The atherosclerotic process of narrowing and hardening of arteries can occur in each artery of the human body although it mainly affects coronary, cerebral, and peripheral arteries (especially those in the lower extremities). ${ }^{2}$ PAOD has a high prevalence in the general population, affecting up to $20 \%$ of persons over 70 years of age. ${ }^{3}$ These numbers indicate that PAOD affects over 27 million individuals in North America and Europe. ${ }^{2}$ The manifestation of PAOD varies from absence of symptoms to tissue loss that may eventually require amputation of an affected limb. PAOD patients commonly present with symptoms of typical intermittent claudication (IC, which means "to limp") in $50 \%-80 \%$ of the cases. ${ }^{2}$ IC is defined as muscle discomfort in a lower limb elicited by exercise and relieved by rest within $10 \mathrm{~min}^{2}$ IC has a significant impact on quality of life. New insights to optimize PAOD (IC) treatment are based on noninvasive conservative as well as minimally invasive strategies. However, restructuring the provided health care system may support even more efficient PAOD treatment. The aim of this article is to introduce an innovative treatment model based on the World Health Organization (WHO) chronic care framework in combination with an optimized multidisciplinary health care chain and the implementation of innovative eHealth (defined by the WHO as the transfer of health resources and health care by electronic means) and mHealth (defined by the WHO as the use of mobile and wireless technologies to support the achievement of health objectives) technologies.

\section{Efficient conservative treatment of PAOD}

Several international guidelines, including the American College of Cardiology/American Heart Association and the Trans-Atlantic Inter-Society Consensus on Management of Peripheral Arterial Disease describe an overall strategy and basic treatment for patients with symptomatic PAOD. ${ }^{2,4}$ Treatment of PAOD should consist of multicomponent therapy of cardiovascular risk reduction through pharmacology interventions and lifestyle coaching and symptomatic treatment. ${ }^{5}$

The first component aims to prevent cardiovascular events (myocardial infarction, stroke) and related morbidity and mortality. The prevalence of cerebrovascular disease in patients with PAOD is approximately $25 \%-50 \% .{ }^{6}$ In a subgroup of patients with severe and symptomatic PAOD a 15 -fold increase in mortality was found. ${ }^{7}$ For IC, a 5-year mortality rate of $19.2 \%$ was described, of which $70 \%$ was due to cardiovascular causes. Cardiovascular risk reduction is important for preventing cardiovascular events (myocardial infarction, stroke) and related morbidity and mortality. The most important modifiable risk factors for atherosclerosis are smoking, hypertension, diabetes mellitus, hyperlipidemia, and obesity. ${ }^{8}$ According to international guidelines, all symptomatic patients with or without a history of cardiovascular disease are generally prescribed antiplatelet therapy in combination with a statin. ${ }^{9}$

The second component aims at relieving symptoms related to PAOD. An initial treatment modality is exercise therapy, first suggested by Erb in $1898 .{ }^{10}$ This approach was supported more than a century later by a Cochrane review. In this review, an overall improvement in maximal walking time with a mean difference of 5.12 minutes (95\% confidence interval [CI]: 4.51-5.72) was found in favor of exercise therapy compared with usual care or placebo. ${ }^{11}$ In daily practice, lack of adequate individual supervision appears to be an important barrier for the initiation and continuation of exercise therapy in patients with PAOD. A Cochrane review by Bendermacher et al and a randomized controlled trial by Nicolai et al showed that supervised exercise therapy (SET) was superior to a unsupervised program. ${ }^{12,13}$ In a cohort study, community-based SET appeared to be as effective as hospital-based SET. ${ }^{13}$ Community-based SET has the advantage of providing a larger capacity of care and is feasible due to the close proximity to the patient's home environment. With this body of evidence, it is clear that SET provided by a physical therapist (PT) in combination with cardiovascular risk management (CVRM) must be offered to all patients with IC.

\section{Current status and problem statement for the Dutch situation}

Unfortunately, only a minority of all newly diagnosed IC patients receive efficient and structured treatment of their disease. The underlying contributing factors impeding fullscale implementation of SET and CVRM programs appear to include:

- Outdated national guidelines

The guidelines of the Dutch Association of General Practitioners and the Dutch Institute for Health Care Improvement guidelines followed by Dutch vascular surgeons are at best ambiguous and do not mention the need of SET. ${ }^{14,15}$ In contrast, the contemporary international guidelines for PAOD explicitly state supervision as a necessary component in exercise therapy.

- Availability of a valid and established SET program

When medical specialists and general practitioners have verified the diagnosis, patients require referral for SET. 
However, whom to refer to? Insights in PTs who provide qualitative SET programs were not available. Transparency and accessibility to quality SET programs are required.

Most of the 23,000 Dutch PTs are inadequately trained according to the Royal Dutch Society for Physical Therapy guidelines for IC. Those who are adequately trained do not always have sufficient experience to provide this form of therapy in the correct manner. Moreover, a SET program has a broader scope than physiotherapy alone. An essential feature of SET is that full attention is paid to existing comorbidity and modifiable lifestyle factors. If these aspects are not addressed appropriately, suboptimal results and loss of resources will occur. Adequately trained PTs should ideally be able to perform individual training, recognize symptoms, provide lifestyle counseling, and monitor medication adherence.

\section{- Reimbursement issues}

A major problem is that the Dutch health care system does not stimulate the use of conservative treatment modalities for patients with IC. Cardiovascular risk management and invasive vascular interventions are fully compensated by the Dutch basic health care insurance. However, for patients with IC, the first 20 treatment sessions of a SET program are not covered under this basic health care insurance (since January 1, 2012) and must be paid by the patient (either directly or through additional insurance). From the 21 st session onwards, all additional treatment sessions given in 1 year are covered by the basic health care insurance. This peculiar reimbursement policy results in a financially driven motivation to be treated by a vascular surgeon with a minimal invasive vascular intervention (eg, angioplasty) or even bypass surgery (both fully reimbursed). This occurs despite the associated risk of morbidity $(<0.5 \%-10 \%)$ and even mortality $(2 \%-3 \%$ for bypass surgery). ${ }^{9}$

- A knowledge gap

- In a recent nationwide survey among Dutch vascular surgeons, approximately $70 \%$ of vascular surgeons believe that coexisting significant cardiopulmonary comorbidity and/or an aorta-iliac stenosis or occlusion are arguments for not referring a patient to a SET program. Recent evidence shows that these arguments are outdated. ${ }^{16}$

- For severe or invalidating IC cases, as subjective as these may be, invasive vascular interventions are performed without awaiting the possible positive effects of a SET program.

\section{Introducing the ClaudicatioNet concept}

To tackle the present shortcomings and optimize the cohesion and collaboration between all stakeholders (including the patient and insurance companies), the ClaudicatioNet concept was launched in The Netherlands in 2011. The goal of ClaudicatioNet is to improve the quality and transparency of PAOD treatment. This system includes an optimal working referral system by stimulating multidisciplinary health care supported with innovative eHealth solutions. The ClaudicatioNet concept aims at nationwide enrollment. Regional networks were created based on the 12 Dutch provinces and local hospital coverage.

\section{The role of a GP or vascular surgeon in the ClaudicatioNet concept}

A regional network was initiated by vascular surgeons to introduce the concept to apprehensive health care professionals together with local, enthusiastic, and motivated PTs. All health care professionals must be familiarized with the concept and their respective roles. The first step in the patient's treatment strategy is determining the diagnosis of IC by a physician (GP or vascular surgeon). Subsequently, a GP or a vascular surgeon should initiate a treatment strategy (conservative vs invasive) and support the coordinating health care professional (CHP) in complex cases.

\section{The role of a coordinating health care professional in the ClaudicatioNet concept}

A CHP (eg, GPs and vascular surgeons or their supporting staff) is pivotal within the ClaudicatioNet concept and the management of the multidisciplinary treatment of PAOD patients. As can be seen in Figure 1, the collaboration between the CHP, PT, and patient is essential in the ClaudicatioNet model. The supporting staff member of a physician (vascular nurse, GP practice assistant) accepts the role of CHP. The CHP functions as a care manager coordinating a patient's CVRM and SET and assesses the availability of high-quality care in the vicinity of the patient. Furthermore, the CHP collaborates closely with the PTs in the regional network, overseeing the quality of care provided. Ultimately, these CHPs must embrace, participate, and manage their regional network based on treatment results and opinions of treated patients. Auxiliary treatment by specialized health care professionals (CVRM prevention 


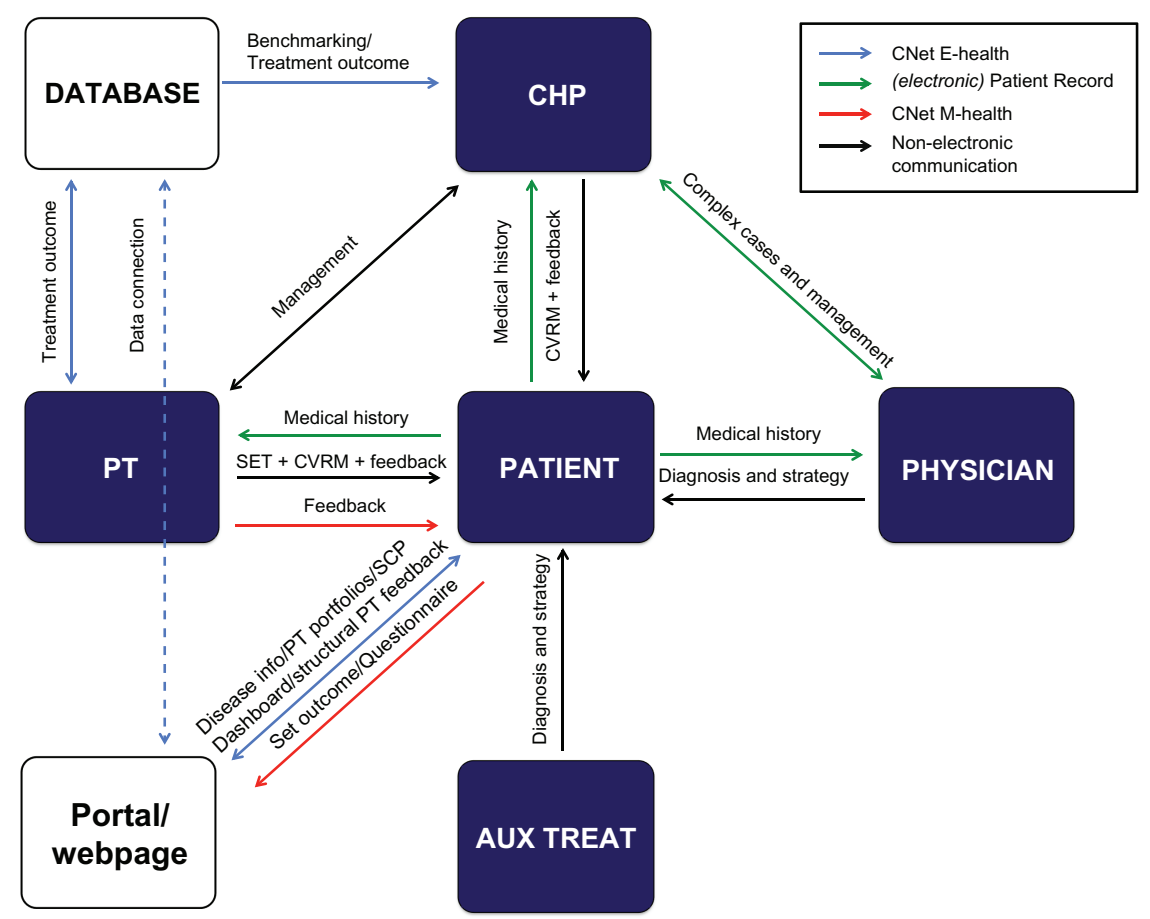

Figure I Schematic overview of role models within the ClaudicatioNet (CNet) Concept.

Abbreviations: PT, physical therapist; CHP, coordinating health care professional; AUX TREAT, auxiliary treatment professional; SET, supervised exercise treatment; CVRM, cardiovascular risk management; $\mathrm{T}$, treatment; SCP, social community platform.

and/or diabetic treatment teams) can support the CPH by providing specialized care for the patient.

\section{The role of a specialized PT in the ClaudicatioNet concept}

PTs must understand the 3-year specialization program. At the forefront of the treatment program are specialized PTs who provide SET and, if applicable, stimulate lifestyle changes and medication adherence. PTs are required to treat patients with contemporary SET programs. To guarantee quality, ClaudicatioNet provides a professional development program for specialization in the field of PAOD. This program is complementary to existing specializations in chronic illness or cardiopulmonary rehabilitation (eg, COPD, heart failure). Apart from acquiring a sound theoretical base of knowledge, PTs gain practical knowledge by increasing their experience by regularly treating patients with PAOD.

\section{The role of the patient}

\section{in the ClaudicatioNet concept}

The patient fulfills an important role in the concept by delivering objective as well as subjective feedback of provided care to all stakeholders (Figure 1). SET outcomes such as maximal walking distance, functional walking distance, quality of life, and information on smoking cessation and medication compliance are useful in evaluating the quality of provided care.

\section{Creating an efficient referring system}

A 3-month SET program that actually starts 2 weeks before a 3-month evaluation appointment precludes evaluation of a first-line conservative treatment approach and is therefore inefficient. Delaying the initiation of a SET program occurs frequently, both due to patient or PT factors (holidays, waiting lists, postponing the first appointment by the patient, etc).This can be prevented by a web-based referral system (automated patient-PT allocation and software that locates and notifies the PT closest to the patient's home). The PT should contact the patient within a limited time frame (for example, 2 working days) and initiate the SET program within 5 working days. In case a PT does not make contact with the patient within 2 working days, a nearby PT is notified automatically. The first responder is then awarded with the possibility to treat a patient and subsequent reimbursement.

\section{Quality of provided care}

All PTs can join a regional network; however, they must meet a set of criteria. Completion of the registered course 
"Intermittent Claudication" is obligatory. As well as a well-equipped practice and a broad patient orientation, to recognize and report other limitations. After these primary criteria are met, the PT enters a specialization program and is entered into an online web-based "care-finder" directory. This website is used by patients as well as CPHs to choose a PT based on aspects such as location (address or postal code) and personal portfolio data (Figure 1). During this three-year specialization program, PTs must comply with a progressive set of criteria to ensure participation. If these criteria are not met, visibility on the care-finder ends leading to interruption of referral.

\section{Participation criteria}

1. The PT delivers care according to up-to-date evidence based guidelines in PAOD SET treatment.

2. The PT provides standardized feedback of results, motivation, and medication adherence to the CHP.

3. The PT maintains correct patient administration, including outcome and process indicators of all patients.

4. The PT actively participates in a life-long learning in the field of peripheral arterial disease and concomitant disease. Fifteen hours of CNet accredited courses annually and presence (every other year) at the yearly organized ClaudicatioNet congress are mandatory.

5. The PT maintains an up to date web-based personal portfolio (http://www.claudicationet.nl). In this profile, all items of PT specialization progress can be visualized. Items such as attendance at additional relevant courses, on-site visitation reports, contact information, a photograph (recognition of caregiver), as well as space for some personal (ambition) statements are registered in this portfolio.

\section{Transparency of provided care}

ClaudicatioNet improves the transparency of provided care by creating clear and extensive portfolios on the care-finder website. Portfolios will be updated with real-time outcome information as soon as a connection is made between the national ClaudicatioNet database and currently existing patient managing software. As of 2013, patients and CHPs will have access to this data through a user-friendly dashboard in order to visualize a clear overview of outcome results (Figure 1). This will also allow patients to gain direct feedback on their own results through a patient's personal web portal (http://www.etalagebenen.nl).

CHPs will be able to effectively monitor the progress of their patients and, more importantly, monitor outcome results of their own regional network by comparing these outcomes with a nationwide benchmark. The development of this innovative online feedback system enhances quality control management and strengthens the multidisciplinary health care chain. PTs with outstanding results can easily be identified and showcased. Others with suboptimal results will be stimulated to learn from these colleagues or, if needed, removed from the care-finder directory.

\section{Improving patient empowerment}

Stimulating self-management causes the patient to learn and become more engaged in his own health process. This is needed in PAOD treatment because of the importance of lifestyle changes and a proactive attitude. Implementation of eHealth and mHealth technology is promising and may significantly reduce health care costs.

\section{eHealth solutions}

Structural feedback from patients regarding the quality of provided SET through a simple short questionnaire offers tremendous possibilities (Figure 1). For example, patients will be asked about their opinions of involved caregivers (GP, VS, CPH, and PT) and the provided SET program. This information is then compared and analyzed with objective SET outcome results (maximal walking distance, functional walking distance, quality of life surveys).

To improve patient responsibility in the treatment of PAOD, some of the numerous SET sessions can be utilized to introduce patients to the personal web-portal. Patients gain access to an online community specifically geared towards PAOD patients. This portal brings together a wealth of information for patients through highly accessible online resources. The website will have two components, including a public domain and a personal portfolio. The public section will contain general disease information on PAOD and information regarding treatment modalities (Figure 1). This website will include a social community platform, where patients can view public profiles and engage with peers, as well as a notice board where patients can post messages for other network participants (Figure 1). Patients can exchange experiences, knowledge, or get in touch with each other (by chat, forums, or through a video call connection). The personal section allows access to a patient journal (with outcome results registered by their PT), their treatment plan, and questionnaires in order to monitor, evaluate, and benchmark the care provided. It also allows synchronous and asynchronous contact with health care providers through modalities as mail, chat, and video call. 


\section{mHealth solutions}

The omnipresence of mobile technology offers unique opportunities for the health care industry. Mobile technology has the potential to make health care better, faster, cheaper, and more accessible. mHealth can improve the quality of care in that it helps patients and clinicians gain a better understanding of disease through the wealth of personalized information generated by remote health monitoring devices and applications. This technology increases access and efficiency by allowing patients and physicians to monitor and analyze (real-time) health data and empowering patients to learn and become engaged in their health process. Lastly, it can significantly reduce health care costs by eliminating unnecessary treatment and facilitating patients to self-manage their condition through automated and highly personal tools.

ClaudicatioNet is developing a mobile application to augment the patient personal digital portfolio. The 'WalkMate' app is an innovative application with several features to help reinforce self-management and patient empowerment through peer support, gamification, and telecoaching. The application features an ambient location radar in order to search for fellow patients or volunteers within the WalkMate network, a prerecorded audio coach for walking advice and stimulation, global positioning system tracking device to register walking distances and speed, live coaching by (schooled) peers, and coach (PT) support through an audio/video call system (Figure 1). The diversity and the ability to personalize the available functions, the presence of virtual and live coaching, and the facilitation of social interaction and support make this application a powerful tool in conjunction with SET. Ultimately, outcome parameters of the WalkMate application will be incorporated into the personal patient portfolio on the personal domain of portal website (http://www.etalagebenen.nl). This application will go live in 2013.

\section{Conclusion}

Physicians, CHP's, PTs and, most of all, patients will benefit from participation in a regional ClaudicatioNet network. By referring patients to ClaudicatioNet PTs, health care professionals are guaranteed transparent and standardized high-quality treatment. Through ClaudicatioNet, all caregivers are collaboratively able to provide the best standards of practice. In time, other treatment goals such as lifestyle counseling and monitoring medication adherence will be taken up by all involved caregivers to extend the possibilities of SET.
Optimizing the concept will stimulate the effectiveness of a multidisciplinary health care chain that is currently required. An economic recession in combination with an aging population will cause associated increase of costs. The ClaudicatioNet concept is likely to result in more efficient and cost-effective PAOD treatment. First, this system will improve the quality of provided SET by offering PTs a controlled specialization program. Second, the system will introduce a free-market principle in health care by creating transparency of provided care through the usage of objective outcome parameters. Furthermore, SET extended with a focus on cardiovascular risk reduction in a high-risk population should be effective because of the reduction in morbidity and mortality caused by diseases with a cardiovascular origin. Cost-effectiveness is achieved because a SET program will substitute or at least postpone expensive invasive vascular interventions. Encouraging patient empowerment by using innovative eHealth and mHealth solutions will create an online community, thereby informing and connecting fellow patients.

\section{Acknowledgments}

The authors are grateful for the assistance in writing of Janno Barlage, bc CEO of Fysio24, Nicole Verhofstad, PhD, as well as Daniëlle van Dalen, MSc, both from our department of vascular surgery, Catharina Hospital, Eindhoven, The Netherlands.

\section{Disclosure}

The authors report no conflicts of interest in this work.

\section{References}

1. Rapport De zorg: hoeveel extra is het ons waard? (Dutch Government Report: healthcare, how much is it worth extra?) Dutch Ministry of Health Welfare and Sports. June 12, 2012. (Dutch)

2. Norgren L, Hiatt WR, Dormandy JA, Nehler MR, Harris KA, Fowkes FG; TASC II Working Group. Inter-Society Consensus for the Management of Peripheral Arterial Disease (TASC II). J Vasc Surg. 2007;45 Suppl S: S5-S67.

3. Selvin E, Erlinger TP. Prevalence of and risk factors for peripheral arterial disease in the United States: results from the National Health and Nutrition Examination Survey, 1999-2000. Circulation. 2004;110(6):738-743.

4. Hirsch AT, Haskal ZJ, Hertzer NR, et al. ACC/AHA 2005 Practice Guidelines for the management of patients with peripheral arterial disease (lower extremity, renal, mesenteric, and abdominal aortic): a collaborative report from the American Association for Vascular Surgery/Society for Vascular Surgery, Society for Cardiovascular Angiography and Interventions, Society for Vascular Medicine and Biology, Society of Interventional Radiology, and the ACC/AHA Task Force on Practice Guidelines (Writing Committee to Develop Guidelines for the Management of Patients With Peripheral Arterial Disease): endorsed by the American Association of Cardiovascular and Pulmonary Rehabilitation; National Heart, Lung, and Blood Institute; Society for Vascular Nursing; TransAtlantic Inter-Society Consensus; and Vascular Disease Foundation. Circulation. 2006;113(11):e463-e654. 
5. Lauret GJ, van Dalen DC, Willigendael EM, et al. Supervised exercise therapy for intermittent claudication: current status and future perspectives. Vascular. 2012;20(1):12-19.

6. Criqui MH. Systemic atherosclerosis risk and the mandate for intervention in atherosclerotic peripheral arterial disease. Am J Cardiol. 2001;88(7B):43J-47J.

7. Criqui MH, Langer RD, Fronek A, et al. Mortality over a period of 10 years in patients with peripheral arterial disease. $N$ Engl J Med. 1992;326(6):381-386.

8. Dormandy J, Heeck L, Vig S. Predictors of early disease in the lower limbs. Semin Vasc Surg. 1999;12(2):109-117.

9. Heart Protection Study Collaborative Group. MRC/BHF Heart Protection Study of cholesterol lowering with simvastatin in 20,536 high-risk individuals: a randomised placebo-controlled trial. Lancet. 2002;360(9326):7-22.

10. Erb W. About intermittent walking and nerve disturbances due to vascular disease [Uber das "intermitterende Hinken" und adere nervose Storungen in Folge von Gefasserkrankungen]. Deutsch Z Nervenheilk. 1898;13:1-76.

11. Watson L, Ellis B, Leng GC. Exercise for intermittent claudication. Cochrane Database Syst Rev. 2008;4:CD000990.
12. Bendermacher BL, Willigendael EM, Teijink JA, Prins MH. Supervised exercise therapy versus non-supervised exercise therapy for intermittent claudication. Cochrane Database Syst Rev. 2006;19(2):CD005263.

13. Nicolai SP, Teijink JA, Prins MH; Exercise Therapy in Peripheral Arterial Disease Study Group. Multicenter randomized clinical trial of supervised exercise therapy with or without feedback versus walking advice for intermittent claudication. J Vasc Surg. 2010;52(2): 348-355.

14. Bartelink M, Stoffers H, Boutens E, Hooij J, Kaiser V, Boomsma L. NHG-standaard Perifeer arterieel vaatlijden [Guideline peripheral arterial disease from the Dutch General Practioners Society]. Huisarts en Wetenschap. 2003;46:848-858.

15. Vahl AC, Reekers JC. Richtlijn diagnostiek en behandeling van arterieel vaatlijden van de onderste extremiteit. (The guideline, Diagnosis and treatment of peripheral artery disease of the lower extremities.) Ned Tijdschr Geneeskd. 2005 July 23;149(30). (Dutch)

16. Lauret GJ, van Dalen HC, Hendriks HJ, et al. When is supervised exercise therapy considered useful in peripheral arterial occlusive disease? A nationwide survey among vascular surgeons. Eur J Vasc Endovasc Surg. 2012;43(3):308-312.
Journal of Multidisciplinary Healthcare

\section{Publish your work in this journal}

The Journal of Multidisciplinary Healthcare is an international, peerreviewed open-access journal that aims to represent and publish research in healthcare areas delivered by practitioners of different disciplines. This includes studies and reviews conducted by multidisciplinary teams as well as research which evaluates the results or conduct of such teams or

\section{Dovepress}

healthcare processes in general. The journal covers a wide range of areas and welcomes submission from practitioners at all levels, from all over the world. The manuscript management system is completely online and includes a very quick and fair peer-review system. Visit http://www.dovepress.com/testimonials.php to read real quotes from published authors. 